\title{
Clinical Characteristics and Clinical Outcomes of Patients with Heart Failure Who Receive Public Assistance in Japan
}

\section{Shingo Watanabe, MD*}

The Department of Cardiology, Tokyo Yamate Medical Center, Japan

*Corresponding author: Shingo Watanabe, The Department of Cardiology, Tokyo Yamate Medical Center, 3-22-1 Hyakunincho, Shinjuku-ward, 169-0063, Tokyo, Japan, Tel: +81-03-3364-0251, Fax: +81-03-5330-2516

\begin{abstract}
Background: The number of patients with heart failure (HF) is increasing, which is a socio-economic problem.

Past overseas studies have reported that low-income patients with HF do not receive adequate treatment for financial reasons and have poor clinical outcomes. In Japan, medical expenses are free if they receive public assistance called Seikatsu hogo (PA), and there is no disadvantage to low-income patients regarding medical expenses. The purpose of this study is to investigate the clinical characteristics of patients with HF who receive PA in Japan.
\end{abstract}

Methods: We enrolled 301 patients who were admitted to our hospital for the congestive HF. We divided patients into groups of patients receiving PA (PA group, $N=51$ ) and groups of patients not receiving PA (Non-WPA group, $\mathrm{N}=250$ ). We compared patient clinical characteristics at admission, and the incidence of one-year cardiovascular events (cardiac death, readmission for HF) in both groups.

Results: The PA group was significantly younger $(71.1 \mathrm{y} \pm$ 12.7 vs. $79.9 y \pm 12.4 \mathrm{P}<0.001)$ and more smokers $(62.7 \%$ vs. $35.6 \% \mathrm{P}<0.001$ ) than the Non-PA group.

The left ventricular ejection fraction was significantly lower in the PA group than in the Non-PA group $(34.6 \% \pm 17.9 \mathrm{vs}$ $43.0 \% \pm 18.7 \mathrm{P}=0.04$ ).

There was no significant difference in mortality between the two groups. The incidence of readmissions for HF was significantly higher in the PA group than in the Non-PA group $(33.3 \%$ vs. $18.2 \% \mathrm{P}=0.02)$.

Conclusion: Patients with HF who received public assistance had a poor cardiac function, and the incidence of readmission due to HF was high.

\section{Keywords}

Public assistance, Heart failure, Low-income patients, Socioeconomic, Universal insurance system

\section{Introduction}

The number of patients with heart failure (HF) has increased in recent years, and it has become a social and economic problem [1]. Past studies have shown that low-income patients have a higher incidence of HF [2]. Past studies have also reported poor outcomes for patients with HF in low-income countries and lowincome regions $[3,4]$. It is believed that this is due to the poor quality of medical care available to low-income patients.

On the other hand, even in Taiwan, which is a universal insurance system, it is known that lowincome HF patients have a high mortality rate and a high incidence of cardiovascular events [5]. The authors point out that the lack of evidence-based guidelinedirected medical therapy for HF in low-income patients due to geographical or poverty factors are associated with poor prognosis in low-income HF patients.

Like Taiwan, Japan has introduced a universal health insurance system for medical care. In Japan, low-income people are publicly subsidized for the minimum living expenses by receiving public assistance called Seikatsu hogo. In addition, medical expenses are free for patients receiving public assistance. Japan has a system in which low-income patients have no financial disadvantage in receiving medical care.

In recent years, the number of public assistance recipients has been increasing, which has become a socio-economic problem because the payment of living expenses and medical expenses to public assistance recipients is paid at national expense [6].

Citation: Watanabe S (2021) Clinical Characteristics and Clinical Outcomes of Patients with Heart Failure Who Receive Public Assistance in Japan. Int Arch Cardiovasc Dis 5:047. doi.org/10.23937/26433966/1710047

Accepted: November 23, 2021: Published: November 25, 2021

Copyright: (C) 2021 Watanabe S. This is an open-access article distributed under the terms of the Creative Commons Attribution License, which permits unrestricted use, distribution, and reproduction in any medium, provided the original author and source are credited. 
There is no study in the literature that investigated the clinical characteristics of HF patients receiving public assistance in Japan.

The aim of our study is to investigate the clinical characteristics and clinical outcomes of HF patients receiving public assistance in Japan.

\section{Methods}

\section{Study populations}

This study is a single-center, retrospective cohort study. The subjects were 337 Japanese patients with their first congestive HF who were admitted to our cardiac care unit from January 2016 to December 2018. Patients who were diagnosed with congestive HF based on medical records were included in the study. Patients with a history of hospitalization for HF were excluded from the study. 36 patients who could not be followed up at our hospital after discharge were excluded from the study. The remaining 301 people were included in the study.

Patients were divided into a group receiving public assistance (PA group $N=51$ ) and a group not receiving public assistance (Non-PA group $N=250$ ). In both groups, the patient background, blood test findings at admission, echocardiographic cardiac function (left ventricular ejection fraction, left ventricular enddiastolic diameter, left atrial volume index, E, and A) at admission, and clinical outcomes for one year after admission for heart failure were investigated.

Written informed consent was obtained from all of the patients and our local institutional review board approved the study protocol. The study was carried out according to the Declaration of Helsinki.

\section{Clinical outcomes}

The clinical outcome of this study was set to the incidence of cardiovascular death and readmission (more than once) due to HF for one year after admission for HF.

\section{Echocardiography}

Transthoracic echocardiography (TTE) was performed within 2 days after the admission. Left ventricular ejection fraction (LVEF) was calculated by a modified version of the Simpson method.

\section{Statistical analysis}

Data were summarized using mean and SD for continuous variables and proportions for categorical variables. Group differences for continuous values were assessed using unpaired two-tailed t-test. Group differences for nominal variables were compared using $\chi^{2} . P<0.05$ was considered statistically significant. Statistical analysis was performed using Bell Curve for Excel (Social Survey Research Information Co., Ltd).

Table 1: Baseline characteristics.

\begin{tabular}{|c|c|c|c|}
\hline & PA group $(N=51)$ & Non-PA group $(\mathrm{N}=250)$ & P Value \\
\hline Age (y) & $71.1 \pm 12.7$ & $79.9 \pm 12.4$ & $P<0.001$ \\
\hline Male $(\%)$ & 58.8 & 57.2 & $P=0.83$ \\
\hline BMI $\left(\mathrm{kg} / \mathrm{m}^{2}\right)$ & $22.5 \pm 3.38$ & $22.1 \pm 4.50$ & $P=0.50$ \\
\hline $\mathrm{DM}(\%)$ & 35.3 & 33.6 & $P=0.81$ \\
\hline $\mathrm{HT}(\%)$ & 68.6 & 62.0 & $P=0.37$ \\
\hline DL (\%) & 11.7 & 23.2 & $P=0.07$ \\
\hline Smoking (\%) & 62.7 & 35.6 & $P<0.001$ \\
\hline Medication for DM, HT, and DL (\%) & 49.0 & 68.4 & $P=0.008$ \\
\hline $\mathrm{SBP}(\mathrm{mmHg})$ & $157.1 \pm 43.6$ & $152.8 \pm 35.8$ & $P=0.55$ \\
\hline $\mathrm{BNP}(\mathrm{pg} / \mathrm{ml})$ & $859.3 \pm 683.7$ & $894.2 \pm 603.0$ & $P=0.84$ \\
\hline $\mathrm{TP}(\mathrm{mg} / \mathrm{dl})$ & $6.7 \pm 0.74$ & $6.67 \pm 0.74$ & $P=0.69$ \\
\hline Total-cholesterol (mg/dl) & $173.0 \pm 44.4$ & $171.2 \pm 42.6$ & $P=0.82$ \\
\hline HDL-cholesterol (mg/dl) & $43.3 \pm 15.3$ & $45.1 \pm 14.3$ & $P=0.50$ \\
\hline LDL-cholesterol (mg/dl) & $105.7 \pm 33.4$ & $101.9 \pm 34.4$ & $P=0.51$ \\
\hline $\mathrm{TG}(\mathrm{mg} / \mathrm{dl})$ & $95.9 \pm 52.6$ & $91.7 \pm 49.4$ & $P=0.64$ \\
\hline Creatinine (mg/dl) & $1.67 \pm 1.76$ & $1.45 \pm 0.94$ & $P=0.40$ \\
\hline $\mathrm{UA}(\mathrm{mg} / \mathrm{dl})$ & $7.27 \pm 2.27$ & $7.31 \pm 2.34$ & $P=0.92$ \\
\hline $\mathrm{HbA1c}(\%)$ & $6.53 \pm 1.39$ & $6.18 \pm 0.96$ & $P=0.15$ \\
\hline WBC $(/ \mu \mathrm{l})$ & $8250 \pm 4077$ & $9231 \pm 4730$ & $P=0.14$ \\
\hline $\mathrm{Hb}(\mathrm{g} / \mathrm{dl})$ & $12.6 \pm 2.43$ & $11.9 \pm 2.22$ & $P=0.08$ \\
\hline
\end{tabular}

BMI: Body Mass Index; BNP: Brain Natriuretic Peptide; DM: Diabetes Mellitus; DL: Dyslipidemia; Hb: Hemoglobin; HT: Hypertension; HDL-Cho: High Density Lipoprotein Cholesterol; LDL-Cho: Low Density Lipoprotein Cholesterol; SBP: Systolic Blood Pressure; T-Cho: Total Cholesterol; TG: Triglyceride; UA: Serum Uric Acid; WBC: White Blood Cell 


\section{Results}

In terms of baseline characteristics, the PA group was significantly younger $(71.1 \mathrm{y} \pm 12.7 v s .79 .9 \mathrm{y} \pm 12.4 \mathrm{P}$ $<0.001)$ and more smokers $(62.7 \%$ vs. $35.6 \% \mathrm{P}<0.001)$ than the Non-PA group (Table 1). In the PA group, the proportion of patients receiving oral treatment for lifestyle-related diseases (diabetes, hypertension, dyslipidemia) were lower than in the Non-PA group (49.0\% vs. $68.4 \% \mathrm{P}=0.008$ ). There was no significant difference between the two groups in terms of blood tests at admission.

Regarding echocardiographic findings at admission, the left ventricular ejection fraction was significantly lower in the PA group than in the Non-PA group (34.6\% \pm 17.9 vs. $43.0 \% \pm 18.7 \mathrm{P}=0.04)$. The left atrial volume index was significantly higher in the PA group than in the Non-PA group $\left(69.9 \mathrm{ml} / \mathrm{m}^{2} \pm 26.9\right.$ vs. $54.7 \mathrm{ml} / \mathrm{m}^{2} \pm$ $29.0 P=0.03$ ) (Table 2).
There was no significant difference between the two groups in terms of the classification of HF (Table 3).

There was no significant difference between the two groups in terms of oral medication for HF after discharge (Table 4). Almost all patients were taking angiotensin converting enzyme inhibitor or angiotensin II receptor blocker. Half of the patients were taking $\beta$-blockers, and about $30 \%$ were taking mineralocorticoid receptor antagonist.

Regarding cardiovascular events during the first year after discharge, the incidence of cardiac death was not significantly different between the two groups $(7.8 \%$ vs. $6.0 \% \mathrm{P}=0.62$ ). The incidence of readmissions due to $\mathrm{HF}$ was significantly higher in the PA group than in the NonPA group (33.3\% vs. $18.2 \% \mathrm{P}=0.02$ ) (Table 5 ).

\section{Discussion}

Our study showed that HF patients receiving public

Table 2: Transthoracic echocardiographic findings on admission.

\begin{tabular}{|l|l|l|l|}
\hline & PA group $(\mathbf{N}=\mathbf{5 1})$ & Non-PA group $(\mathbf{N}=\mathbf{2 5 0})$ & P Value \\
\hline LVEF $(\%)$ & $34.6 \pm 17.9$ & $43.0 \pm 18.7$ & $\mathbf{P = 0 . 0 4}$ \\
\hline LVDd $(\mathrm{mm})$ & $53.1 \pm 9.46$ & $51.9 \pm 10.6$ & $P=0.56$ \\
\hline LAV index $\left(\mathrm{ml} / \mathrm{m}^{2}\right)$ & $69.9 \pm 26.9$ & $54.7 \pm 29.0$ & $\mathbf{P = 0 . 0 3}$ \\
\hline E $(\mathrm{cm} / \mathrm{s})$ & $78.4 \pm 30.4$ & $77.6 \pm 32.5$ & $P=0.91$ \\
\hline A $(\mathrm{cm} / \mathrm{s})$ & $71.8 \pm 36.6$ & $85.4 \pm 30.1$ & $P=0.28$ \\
\hline
\end{tabular}

LAV Index: Left Atrial Volume Index; Lvdd: Left Ventricular End-Diastolic Diameter; LVEF: Left Ventricular Ejection Fraction

Table 3: The classification of heart failure.

\begin{tabular}{|l|l|l|l|}
\hline & PA group (N = 51) & Non-PA group (N = 250) & P Value \\
\hline HHD (\%) & 25.5 & 18.0 & $P=0.21$ \\
\hline AMI (\%) & 10.8 & 5.6 & $P=0.26$ \\
\hline IHM (\%) & 23.5 & 29.2 & $P=0.41$ \\
\hline DCM (\%) & 13.7 & 7.6 & $P=0.41$ \\
\hline HCM (\%) & 3.9 & 2.4 & $P=0.54$ \\
\hline VHD (\%) & 19.6 & 18.4 & $P=0.84$ \\
\hline
\end{tabular}

AMI: Acute Myocardial Infarction; DCM: Dilated Cardiomyopathy; HCM: Hypertrophic Cardiomyopathy; HHD: Hypertensive Heart Disease; IHD: Ischemic Heart Disease; VHD: Valvular Heart Disease

Table 4: Drug treatment at discharge.

\begin{tabular}{|l|l|l|l|}
\hline & PA group $(\mathbf{N}=\mathbf{5 1})$ & Non-PA group $(\mathbf{N}=250)$ & P Value \\
\hline ACEI or ARB (\%) & 96.0 & 98.4 & $P=0.27$ \\
\hline B blocker (\%) & 45.1 & 45.6 & $P=0.94$ \\
\hline Diuretic (\%) & 68.6 & 72.4 & $P=0.58$ \\
\hline MRA (\%) & 35.3 & 28.9 & $P=0.36$ \\
\hline Statin (\%) & 27.5 & 28.0 & $P=0.93$ \\
\hline
\end{tabular}

ACEl: Angiotensin Converting Enzyme Inhibitor; ARB: Angiotensin II Receptor Blocker; MRA: Mineralocorticoid Receptor Antagonist

Table 5: Clinical outcomes in a year.

\begin{tabular}{|l|l|l|l|}
\hline & PA group $\mathbf{( N = 5 1 )}$ & Non-PA group $(\mathbf{N}=\mathbf{2 5 0})$ & P Value \\
\hline Cardiac Death & 7.8 & 6.0 & $P=0.62$ \\
\hline Readmission due to heart failure & 33.3 & 18.2 & $\mathbf{P = 0 . 0 2}$ \\
\hline
\end{tabular}


assistance in Japan were characterized by a younger age of onset, poor cardiac function at admission to $\mathrm{HF}$, and a higher rate of readmissions due to HF. In Japan, medical cost for patients receiving public assistance is covered by national funds. It is expected that the number of $\mathrm{HF}$ patients will increase in Japan in the future [7], and it is socio-economically important to improve the clinical outcome of HF patients receiving public assistance.

A US study also reported poor clinical outcomes in low-income heart failure patients. In the United States, poor treatment of low-income heart failure patients for financial reasons is considered to be a major cause of poor clinical outcomes [2].

Our study found interesting results that even patients with no financial disadvantage in terms of medical costs had poor clinical outcomes in low-income heart failure patients.

It has been reported that low-income HF patients have many cardiovascular events such as death and readmission due to HF in Taiwan, which has a universal insurance system as in Japan [5]. The authors point out that low-income earners live in rural areas and suburbs and are not properly treated due to geographical factors. Our hospital is located in the urban area of Shinjukuward, Tokyo, and there is no disadvantage in terms of medical access. In addition, there was no significant difference in the resume of $\mathrm{HF}$ treatment between the PA group and the Non-PA group. In our study, the PA group had more smokers and fewer patients were treated for lifestyle-related diseases such as hypertension, diabetes, and dyslipidemia before hospitalization for HF. In addition, the LVEF at admission was significantly lower in the PA group than in the non-PA group. These were thought to have resulted in poor clinical outcomes for HF. It is necessary to promote health examinations to public assistance recipients and to educate them about smoking cessation and management of lifestylerelated diseases.

Previously, we also reported on the clinical characteristics of patients with acute myocardial infarction who are receiving welfare benefits in Japan [8]. The study showed that patients receiving life protection were more likely to develop ventricular arrhythmias during the acute phase of acute myocardial infarction and had poor cardiac function during the chronic phase. This study also showed that welfare recipients had a higher prevalence of smoking before the onset of myocardial infarction and were younger. Based on this result, it was considered necessary to intervene in the management of lifestyle-related diseases of welfare recipients from an early stage before the onset of cardiovascular events.

\section{Limitation}

Our study is a single-center study and facility bias needs to be considered. Our hospital is located in
Shinjuku-Ward, Tokyo, in an urban area of Japan. In the suburbs and rural areas, the characteristics of public assistance recipients are considered to be different from those in urban areas, and our research results cannot be directly applied to non-urban areas.

\section{Conclusion}

Patients with $\mathrm{HF}$ who received public assistance had poor cardiac function at the time of admission, and the incidence of readmission due to $\mathrm{HF}$ was high. For public assistance recipients, it was considered necessary to provide lifestyle-related guidance such as smoking cessation and diet therapy and intervention for lifestylerelated disease treatment before the onset of HF.

\section{Acknowledgement}

The Authors declares that there is no conflict of interest.

\section{Funding}

This research received no grant from any funding agency in the public, commercial or not-for-profit sectors.

We would like to thank Dr. Yukiko Watanabe for her advice.

\section{References}

1. Ziaeian B, Fonarow GC (2016) Epidemiology and aetiology of heart failure. Nat Rev Cardiol 13: 368-378.

2. Akwo EA, Kabagambe EK, Wang TJ, Harrell Jr FE, Blot WJ, et al. (2017) Heart failure incidence and mortality in the southern community cohort study. Circ Heart Fail 10: e003553.

3. Dewan P, Rorth R, Jhund PS, Ferreira JP, Zannad F, et al. (2019) Income inequality and outcomes in heart failure: $A$ global between-country analysis. JACC Heart Fail 7: 336346 .

4. Kind AJH, Jencks S, Brock J, Yu M, Bartels C, et al. (2014) Neighborhood socioeconomic disadvantage and 30-day rehospitalization: $A$ retrospective cohort study. Ann Intern Med 161: 765-774.

5. Hung C-L, Chao T-F, Su C-H, Liao J-N, Sung K-T, et al. (2021) Income level and outcomes in patients with heart failure with universal health coverage. Heart 107: 208-216.

6. Hayashi M (2011) The effects of medical factors on transfer deficits in public assistance in japan: A quantile regression analysis. Int J Health Care Finance Econ 11: 287-307.

7. Okura Y, Ramadan MM, Ohno Y, Mitsuma W, Tanaka K, et al. (2008) Impending epidemic: Future projection of heart failure in japan to the year 2055. Circ J 72: 489-491.

8. Watanabe S, Usui M (2021) Clinical features of st-segment elevation myocardial infarction in patients receiving welfare public assistance in urban area of japan. J Cardiol 77: 404407. 